Chirurg 2021 $\cdot 92: 168$

https://doi.org/10.1007/s00104-020-01335-z

Angenommen: 9. Dezember 2020

Online publiziert: 8. Januar 2021

(c) Springer Medizin Verlag GmbH, ein Teil von Springer Nature 2021

\section{Originalpublikation}

Veld JV, Amelung FJ, Borstlap WAA et al (2020) Decompressing Stoma as bridge to elective surgery is an effective strategy for leftsided obstructive colon cancer: a national, propensity-score matched study. Ann Surg 272:738-743

Hintergrund. Notfallmäßige Resektionen bei maligner linksseitiger Kolonobstruktion (MLKO) gehen insbesondere bei älteren Patienten mit einer hohen postoperativen Letalität und Morbidität einher. Alternative Konzepte bestehen in der Dekomprimierung des Kolons in der Akutsituation mittels endoskopischer Platzierung eines selbstexpandierenden Metallstents (SEMS) oder durch die Anlage eines Stomas. Ziel dieser Verfahren ist es, eine elektive onkologische Resektion unter optimierten klinischen Bedingungen zu ermöglichen („bridge to surgery“, BTS). Die vorgestellte Studie vergleicht die Anlage eines dekomprimierenden Stomas (DS) mit der Notfallresektion (NR).

Material und Methoden. Anhand eines nationalen Registers wurden Patienten erfasst, die zwischen 2009 und 2016 in 75 holländischen Kliniken aufgrund einer MLKO ein DS oder eine NR in kurativer Intention erhielten. Von insgesamt 2048 ausgewählten Patienten wurden nach 1:2 "propensity score matching“ 236 Patienten mit DS und 472 Patienten mit NR in die Studie eingeschlossen. Primärer Endpunkt war die 90-Tage-Letalität, sekundärer Endpunkt das 3-JahresGesamtüberleben sowie die Rate an dauerhaften Stomata.

\title{
Dekomprimierendes Stoma vs. Notfallresektion bei maligner linksseitiger Kolonobstruktion
}

Ergebnisse. Die 90-Tage-Letalitätsrate war nach DS signifikant niedriger als nach NR $(1,7 \%$ vs. $7,2 \%, p=0,006)$. In einer Subgruppenanalyse zeigte sich dieser Effekt insbesondere bei Patienten $\geq 70$ Jahre $(3,5 \%$ vs. $13,7 \%, p=0,027)$. Patienten, die in der Akutsituation ein DS erhielten, hatten ein signifikant besseres 3-Jahres-Gesamtüberleben (79,4\% vs. 73,3\%, Hazard Ratio 0,36) und bekamen seltener ein dauerhaftes endständiges Stoma $(23,4 \%$ vs. $42,4 \%, p<0,001)$. Nach BTS mit DS erfolgten im elektiven Intervall signifikant mehr laparoskopische Resektionen (56,8\% vs. 9,2\%, $p<0,001)$. Zudem wurden häufiger primäre Anastomosen angelegt $(88,5 \%$ vs. $40,7 \%, p<0,001)$. Hinsichtlich der Gesamtkomplikationsrate, der Lokalrezidivrate und des krankheitsfreien Überlebens fanden sich keine signifikanten Unterschiede in den beiden Gruppen.

Diskussion und Fazit. In der Literatur gibt es bislang keine überzeugende Evidenz, die eindeutig Vorteile des DS gegenüber der NR belegt. Zu dieser Thematik erfolgte lediglich eine randomisierte Studie, welche keinen Unterschied in der Letalität fand [1]. Des Weiteren wird häufig angeführt, dass die Stomarückverlagerung immer eine zusätzliche Operation mit eigener Morbidität bedeutet. Allerdings kann die Kontinuität bereits im Rahmen der elektiven Resektion wieder hergestellt werden, wenn das DS nahe an der Tumorlokalisation angelegt wird. Bei der BTS-Strategie mit DS handelt es sich um einen technisch einfach durchzuführenden Eingriff, der auch in der Notfallsituation problemlos minimal-invasiv erfolgen kann. Dies ist insofern auch von
Bedeutung, da die notwendige endoskopische Expertise zur Anlage eines SEMS nicht in jeder Klinik vorhanden ist, und die onkologischen Risiken durch Mikroperforationen des Stents noch nicht abschließend geklärt sind. In einer rezent veröffentlichten Arbeit fand sich zudem ein nicht signifikanter Trend $\mathrm{zu}$ einem besseren Gesamtüberleben nach DS vs. SEMS [2].

\section{Korrespondenzadresse}

\section{Prof. Dr. M. Anthuber}

Klinik für Allgemein-, Viszeral- Transplantationschirurgie, Klinikum Augsburg Stenglinstr. 2, 86156 Augsburg, Deutschland matthias.anthuber@klinikum-augsburg.de

Interessenkonflikt. A. Straßmüller und M. Anthuber geben an, dass kein Interessenkonflikt besteht.

\section{Literatur}

1. Kronberg O (1995) Acute obstruction from tumour in the left colon without spread. A randomized trial of emergency colostomy versus resection. Int J Colorectal Dis 10:1-5

2. Veld JV, Amelung FJ, Borstlap WAA et al (2020) Comparison of decompressing stoma vs. stent as a bridge to surgery for left-sided obstructive colon cancer. JAMA Surg 155:206-215 